\title{
Stress measurement using area detectors. A theoretical and experimental comparison of different methods in ferritic steel using a portable x-ray apparatus
}

\author{
J. Ramirez-Rico ${ }^{1, *}$, S.-Y. Lee ${ }^{2}$, J.J. Ling ${ }^{2}$ and I. C. Noyan ${ }^{2}$ \\ ${ }^{1}$ Fisica de la Materia Condensada-ICMS, Universidad de Sevilla-CSIC, 41012 \\ Sevilla, Spain. \\ ${ }^{2}$ Applied Physics and Applied Mathematics, Columbia University, New York, NY \\ 10027 \\ * Corresponding author: jrr@us.es
}

\begin{abstract}
Using area detectors for stress determination by diffraction methods in a single exposure greatly simplifies the measurement process and permits the design of portable systems without complex sample cradles or moving parts. An additional advantage is the ability to see the entire or a large fraction of the Debye ring and thus determine texture and grain size effects before analysis. The two methods most commonly used to obtain stress from a single Debye ring are the so called $\cos \alpha$ and full-ring fitting methods, which employ least-squares procedures to determine the stress from the distortion of a Debye ring by probing a set of scattering vector simultaneously. The widely applied $\sin ^{2} \psi$ method, in contrast, requires sample rotations to probe a different subset of scattering vector orientations. In this paper we first present a description of the different methods under the same formalism and using a unified set of coordinates that are suited to area detectors normal to the incident beam, highlighting the similarities and differences between them. We further characterize these methods by means of in-situ measurements in carbon steel tube samples, using a portable detector in reflection geometry. We show that, in the absence of plastic flow, the different methods yield basically the same results and are equivalent. An analysis of possible sources of errors and their impact in the final stress values is also presented.
\end{abstract}

\section{Introduction}

Area x-ray detectors allow the recording of residual elastic strain in a material along a set of scattering vectors in a single measurement, so that the number of sample rotations required for determination of the residual stress state is significantly reduced and, in some cases, completely eliminated. This well-known fact formed the basis of traditional 'film' techniques, where Debye Ring(s) from a polycrystalline sample were captured on x-ray sensitive photographic film using laboratory systems [1-3] or portable stress analyzers [4]. A comprehensive review of such 'single-exposure' techniques was given by James and Cohen in 1978 [5]. Within the last decade modern detectors which record and(or) read out incident x-ray intensitites electronically from the cells of a pixellated area detector replaced film. Publications utilizing these new area detectors reported 'new' techniques for the analysis of residual stresses, where terms like ' $X R D^{2}$ : Bidimensional X-Ray Diffraction' [6] or '2-dimensional x-ray diffraction' [7] were introduced. In contrast to the conventional 
$\sin ^{2} \psi$ technique used with point detectors, which requires the measurement of strain in at least two distinct sample orientations for plane stress states $[8,9]$, with area detectors the residual stress can in principle be determined in a single exposure, irrespective of the type of detector, or the terms used to describe it. This advantage, combined with the miniaturization of image plates, x-ray sources and associated reading electronics, have resulted in commercial portable apparatus designed for in-line and field measurement applications, especially for ferrous metals [10]. An additional advantage of an area detector is the ability to see the entire Debye Ring before analysis and thus determine texture and grain size effects [11]. Due to these benefits, area detectors are specially well suited for performing in-situ measurements, either at elevated temperatures or as function of applied stress, i.e. references $[12,13]$.

Different approaches for the determination of the stress state from the information contained in a single Debye exposure exist, such as the $\cos \alpha$ method introduced by Sasaki et. al [14-16] or the direct least squares fitting of the measured strain [17]. Despite the attractiveness of these portable devices in both industrial and scientific applications, there is no current agreement among experts on the most efficient, precise and accurate stress determination formalism.

In this work, we compare the three proposed methods from both theoretical and experimental points of view. A code for the generation of synthetic $2 \mathrm{D}$ x-ray diffraction patterns was developed and used to study the different methods' sensitivity to misalignment, detector calibration parameters as well as scatter in the data. For the experimental assessment we have used a portable x-ray residual stress measurement apparatus ( $\mu$-X360 residual stress analyzer from Pulstec Industrial Co., Ltd.) to determine the residual stress in 1010 Carbon Steel cylindrical tubes in-situ during tensile loading. The measurement conditions, with tight space constraints for the detector, as well as the use of a non-flat specimen, were chosen to approach those of actual applications. Several samples were tested through the proportional limit and the residual stress at various loads was determined from single x-ray exposures, using both the $\cos \alpha$ and the direct least squares fitting methods. Measurements were performed at different sample orientations with respect to the incoming beam to both assess its effect on the calculated stress, as well as to allow us to use the $\sin ^{2} \psi$ technique for comparison. We emphasize that our purpose is comparing the stresses obtained by these techniques to each other, not to a known (applied) stress. The latter case would be testing the accuracy of the techniques. However, portable residual stress measurement devices are easy to misalign during field measurements [10], and if so, will measure the wrong stress value independent of the technique used. Our results show that, for a mis-aligned machine all techniques will be in error by the same amount, and in the case of a perfectly aligned sample all three would yield the correct stress.

\section{Stress determination using area detectors}

X-ray based techniques to determine the (elastic) strain and stress in a material rely on the use of inter-planar spacing in a crystalline material as a built-in strain-gauge [7-9]. By measuring the lattice spacing $d$ in a stressed sample and comparing its value with the lattice spacing $d_{0}$ of a stress-free sample (ideally identical to the stressed one in every other aspect), we obtain the projection of the strain tensor along the scattering vector used in the determination of $d$. Let $(\psi, \phi)$ be the polar angles that determine the scattering vector $\vec{q}_{s}$ in sample coordinates, 


$$
\vec{q}_{S}=\left[\begin{array}{c}
\sin \psi \cos \phi \\
\sin \psi \sin \phi \\
\cos \psi
\end{array}\right]=\left[\begin{array}{l}
q_{1} \\
q_{2} \\
q_{3}
\end{array}\right]
$$

Then the strain measured along the scattering vector at angles $(\psi, \phi)$ can be expressed in terms of the strain components $\epsilon_{i j}$ in sample coordinates as:

$$
\begin{aligned}
\epsilon_{\psi \phi}=\left(d_{\psi \phi}-d_{0}\right) / d_{0}=q_{i} q_{j} \epsilon_{i j}= & \epsilon_{11} \cos ^{2} \phi \sin ^{2} \psi+\epsilon_{12} \sin 2 \phi \sin ^{2} \psi \\
& +\epsilon_{22} \sin ^{2} \phi \sin ^{2} \psi+\epsilon_{33} \cos ^{2} \psi \\
& +\epsilon_{13} \cos \phi \sin 2 \psi+\epsilon_{23} \sin \phi \sin 2 \psi
\end{aligned}
$$

Due to the shallow penetration of x-rays in most materials, typically only near-surface stresses can be measured, where due to boundary conditions the normal stresses vanish to zero at the surface [8]. An additional hypothesis is the absence of steep strain gradients $[18,19]$. If that is not the case, a more detailed treatment is needed [20]. It is then customary to assume a biaxial (or plane stress) stress state so that:

$$
\sigma_{i j}=\left[\begin{array}{ccc}
\sigma_{11} & \sigma_{12} & 0 \\
\sigma_{12} & \sigma_{22} & 0 \\
0 & 0 & 0
\end{array}\right] \Longrightarrow \epsilon_{i j}=\left[\begin{array}{ccc}
\epsilon_{11} & \epsilon_{12} & 0 \\
\epsilon_{12} & \epsilon_{22} & 0 \\
0 & 0 & \epsilon_{33}
\end{array}\right]
$$

We will further assume that the material's elastic behavior can be described as isotropic and homogeneous, so Hooke's law takes the form:

$$
\epsilon_{i j}=\frac{1+\nu}{E} \sigma_{i j}-\delta_{i j} \frac{\nu}{E} \sigma_{k k}
$$

Where Einstein's summation convention is used throughout. With these assumptions eq. (2) simplifies to:

$$
\epsilon_{\psi \phi}=\frac{d_{\psi \phi}-d_{0}}{d_{0}}=\left(\epsilon_{11} \cos ^{2} \phi+\epsilon_{12} \sin 2 \phi+\epsilon_{22} \sin ^{2} \phi-\epsilon_{33}\right) \sin ^{2} \psi+\epsilon_{33}
$$

Or, in terms of stresses:

$$
\frac{d_{\psi \phi}-d_{0}}{d_{0}}=\frac{1+\nu}{E} \sigma_{\phi} \sin ^{2} \psi-\frac{\nu}{E}\left(\sigma_{11}+\sigma_{22}\right)
$$

Where $\sigma_{\phi}=\sigma_{11} \cos ^{2} \phi+\sigma_{12} \sin 2 \phi+\sigma_{22} \sin ^{2} \phi$. Equation (5) is the basis of the $\sin ^{2} \psi$ method, that has been used for over 50 years as the x-ray method of choice for stress determination [8].

Now we need to introduce the goniometer conventions that will relate the orientation of the scattering vector in laboratory (diffractometer) coordinates with those in sample coordinates (Figure 1). We will consider a 2D x-ray area detector oriented so it is normal to the incoming (primary) beam and thus records backscattered beams diffracted from the sample $(2 \theta>\pi / 2)$. Diffracted x-rays will, for a certain family of lattice planes, form cones that will intersect the detector forming circles or Debye rings. The semi-angles of these cones will be defined as $2 \eta=\pi-2 \theta$, and the polar angle along each of the Debye rings will be noted as $\alpha$. Thus, for each point in the detector with coordinates $(x, y)$, the equivalent cone coordinates can be determined using [7]: 


$$
\tan 2 \eta=\frac{\sqrt{\left(x-x_{0}\right)^{2}+\left(y-y_{0}\right)^{2}}}{z_{0}}, \quad \tan \alpha=\frac{x-x_{0}}{y-y_{0}}
$$

Where $\left(x_{0}, y_{0}\right)$ describes the location of the incident x-ray beam in detector coordinates, while $z_{0}$ is the sample to detector distance. The scattering vector corresponding to each point in the Debye ring also resides in a cone, in this case of semi-angle $\eta$. Since each point in the ring corresponds to a different orientation of the scattering vector, there must exist a transformation that relates its coordinates $\vec{q}_{L}$ in the lab system to those $\vec{q}_{S}$ in the sample system.

$$
\vec{q}_{L}=\left[\begin{array}{c}
-\cos \eta \\
-\sin \eta \sin \alpha \\
-\sin \eta \cos \alpha
\end{array}\right]=\left[\begin{array}{c}
-\sin \theta \\
-\cos \theta \sin \alpha \\
-\cos \theta \cos \alpha
\end{array}\right] \Longrightarrow \vec{q}_{S}=\left[\begin{array}{c}
\sin \eta \cos \alpha \\
\sin \eta \sin \alpha \\
\cos \eta
\end{array}\right] \Longrightarrow(\psi, \phi)=(\eta, \alpha)
$$

[Figure 1 about here.]

We will now consider sample rotations $\left(\omega_{0}, \psi_{0}, \phi_{0}\right)$ defined according to Figure 1 . The transformation matrix between the two reference systems is then:

$A=\left[\begin{array}{ccc}-\cos \omega_{0} \sin \psi_{0} \cos \phi_{0}+\sin \omega_{0} \sin \phi_{0} & \sin \omega_{0} \sin \psi_{0} \cos \phi_{0}+\cos \omega_{0} \sin \phi_{0} & -\cos \psi_{0} \cos \phi_{0} \\ -\cos \omega_{0} \sin \psi_{0} \sin \phi_{0}-\sin \omega_{0} \cos \phi_{0} & \sin \omega_{0} \sin \psi_{0} \sin \phi_{0}-\cos \omega_{0} \cos \phi_{0} & -\cos \psi_{0} \sin \phi_{0} \\ -\cos \omega_{0} \cos \psi_{0} & \sin \omega_{0} \cos \psi_{0} & \sin \psi_{0}\end{array}\right]$

And so we can transform the coordinates by simple matrix multiplication.

$$
\vec{q}_{S}=A \vec{q}_{L}=\left[\begin{array}{lll}
a_{11} & a_{12} & a_{13} \\
a_{21} & a_{22} & a_{23} \\
a_{31} & a_{32} & a_{33}
\end{array}\right]\left[\begin{array}{c}
-\cos \eta \\
-\sin \eta \sin \alpha \\
-\sin \eta \cos \alpha
\end{array}\right]=\left[\begin{array}{c}
\sin \psi \cos \phi \\
\sin \psi \sin \phi \\
\cos \psi
\end{array}\right]=\left[\begin{array}{c}
q_{1} \\
q_{2} \\
q_{3}
\end{array}\right]
$$

It is instructive to consider some special cases of sample rotations $\left(\omega_{0}, \psi_{0}, \phi_{0}\right)$ that lead to traditional scattering geometries commonly used in x-ray diffraction experiments.

\subsection{Normal incidence}

We consider now the case where $\omega_{0}=\psi_{0}=\phi_{0}=0$. Now the primary x-ray beam is normal to the sample surface, and thus sample and detector planes are parallel. Now, in sample coordinates the scattering vector is simply:

$$
\vec{q}_{S}=\left[\begin{array}{c}
\cos \alpha \sin \eta \\
\sin \alpha \sin \eta \\
\cos \eta
\end{array}\right]
$$

And thus we have $\psi=\eta, \phi=\alpha$. In this geometry the polar angle $\psi$ is fixed and the azimuthal angle $\phi$ is equivalent to the polar angle along the Debye ring.

\subsection{Conventional $\theta-\theta$ geometry}

In a conventional $\theta-\theta$ experiment, the incident beam makes an angle $\theta$ with the sample surface and if $\psi_{0}=0$ the scattering vector is normal to the sample surface, too. This implies that $\omega_{0}=\eta$. In this geometry, the primary and scattered beam lie in a plane containing the scattering vector when $\psi=0$. In a two dimensional vector, this condition is fulfilled at $\alpha=-\pi / 2$, and we get $\vec{q}_{S}=[001]$. 


\subsection{Arbitrary $\psi_{0}$ tilt with $\omega_{0}=0$}

This is the case most relevant to our purposes, since it describes the geometry in the portable x-ray stress measurement device utilized in this study. Now the scattering vector in sample coordinates can be written in terms of sample rotations and Debye ring coordinates as:

$$
\vec{q}_{S}=\left[\begin{array}{c}
\cos \eta \sin \psi_{0} \cos \phi_{0}+\sin \eta \cos \psi_{0} \cos \phi_{0} \cos \alpha-\sin \eta \sin \phi_{0} \sin \alpha \\
\cos \eta \sin \psi_{0} \sin \phi_{0}+\sin \eta \cos \psi_{0} \sin \phi_{0} \cos \alpha+\sin \eta \cos \phi_{0} \sin \alpha \\
\cos \eta \cos \psi_{0}-\sin \eta \sin \psi_{0} \cos \alpha
\end{array}\right]
$$

We must remember that, in general, $(\psi, \phi) \neq\left(\psi_{0}, \phi_{0}\right)$, and the relationship to perform the transformation $(\eta, \alpha) \rightarrow(\psi, \phi)$ is rather complex. Two special cases can be described at angles $\alpha=0, \pi$, as can be seen in Figure 2: at $\alpha=0$ we have $\psi=\psi_{0}+\eta$, while at $\alpha=\pi$ we have $\psi=\psi_{0}-\eta$. This means that, for this geometry, the points in the Debye ring at $\alpha=0, \pi$ can be used directly in the $\sin ^{2} \psi$ method once the $\eta$ angle is accounted for.

[Figure 2 about here.]

\subsection{The $\cos \alpha$ method}

One of the methods that allows the calculation of stress from a single measurement with an area detector is the $\cos \alpha$ method described by Sasaki et al. and others $[14,21]$ and implemented in commercial instrumentation for residual stress determination. The basic idea is to start from the expression of the scattering vector in sample coordinates in terms of Debye ring coordinates, in the case of arbitrary $\psi_{0}$. For simplicity we will restrict ourselves to the sample-detector geometry depicted in Figure 1, where the incoming x-ray beam is normal to the flat $2 \mathrm{D}$ detector and only a rotation along $S_{2}$ in sample coordinates is allowed. For a stress-free sample the scattering vectors lie along cones with semi-apex angle $\eta=\theta-\pi / 2$ for each set of lattice planes fulfilling Bragg's condition, producing a series of concentric Debye rings. For every point in the detector with coordinates $(x, y)$

$$
\vec{q}_{S}=\left[\begin{array}{c}
\cos \eta \sin \psi_{0}+\sin \eta \cos \psi_{0} \cos \alpha \\
\cos \eta \sin \psi_{0} \sin \phi_{0}+\sin \eta \cos \psi_{0} \sin \phi_{0} \cos \alpha+\sin \eta \cos \phi_{0} \sin \alpha \\
\cos \eta \cos \psi_{0}-\sin \eta \sin \psi_{0} \cos \alpha
\end{array}\right]
$$

The strain projection along $(\eta, \alpha)$ coordinates could be written in terms of the scattering vector and strain components as $\epsilon_{\alpha}=q_{i} q_{j} \epsilon_{i j}$. We will assume plane-stress conditions and consider an elastic, isotropic and homogeneous material such that:

$$
\epsilon_{i j}=\frac{1+\nu}{E} \sigma_{i j}-\delta_{i j} \frac{\nu}{E} \sigma_{k k}
$$

We can substitute to find the strain projection in terms of stress components:

$$
\epsilon_{\alpha}=\frac{1+\nu}{E} q_{i} q_{j} \sigma_{i j}-\frac{\nu}{E} \sigma_{k k}
$$

Now we define parameters $a_{1}, a_{2}$ as:

$$
\begin{aligned}
& a_{1}\left(\phi_{0}\right)=\frac{1}{2}\{[\epsilon(\alpha)-\epsilon(\pi+\alpha)]+[\epsilon(-\alpha)-\epsilon(\pi-\alpha)]\} \\
& a_{2}\left(\phi_{0}\right)=\frac{1}{2}\{[\epsilon(\alpha)-\epsilon(\pi+\alpha)]-[\epsilon(-\alpha)-\epsilon(\pi-\alpha)]\}
\end{aligned}
$$


From here we can now obtain:

$$
\begin{aligned}
& \left.a_{1}\left(\phi_{0}\right)=\frac{1+\nu}{E} \sin 2 \psi_{0} \sin 2 \eta \cos \alpha\left[\sigma_{11}\left(1+\cos 2 \phi_{0}\right)+\sigma_{22}\left(1-\cos 2 \phi_{0}\right)+2 \sigma_{12} \sin 2 \phi_{0}\right)\right] \\
& \left.a_{2}\left(\phi_{0}\right)=\frac{1+\nu}{E} \sin \psi_{0} \sin 2 \eta \sin \alpha\left[\sigma_{22} \sin 2 \phi_{0}-\sigma_{11} \sin 2 \phi_{0}+2 \sigma_{12} \cos 2 \phi_{0}\right)\right]
\end{aligned}
$$

And we now consider the special case of $\phi_{0}=0$ to get:

$$
\begin{aligned}
& a_{1}\left(\phi_{0}=0\right)=\frac{1+\nu}{E} \sigma_{11} \sin 2 \psi_{0} \sin 2 \eta \cos \alpha \\
& a_{2}\left(\phi_{0}=0\right)=2 \frac{1+\nu}{E} \sigma_{12} \sin \psi_{0} \sin 2 \eta \sin \alpha
\end{aligned}
$$

So by plotting the parameters $a_{1}, a_{2}$ as a function of $\cos \alpha$ and $\sin \alpha$ we obtain two linear relationships, the slopes of which will be proportional to $\sigma_{11}$ and $\sigma_{12}$, respectively.

\subsection{Full Debye ring fitting}

For simplicity, let us continue assuming that $\omega_{0}=0$, so the scattering vector can still be written as:

$$
\vec{q}_{S}=\left[\begin{array}{c}
\cos \eta \sin \psi_{0} \cos \phi_{0}+\sin \eta \cos \psi_{0} \cos \phi_{0} \cos \alpha-\sin \eta \sin \phi_{0} \sin \alpha \\
\cos \eta \sin \psi_{0} \sin \phi_{0}+\sin \eta \cos \psi_{0} \sin \phi_{0} \cos \alpha+\sin \eta \cos \phi_{0} \sin \alpha \\
\cos \eta \cos \psi_{0}-\sin \eta \sin \psi_{0} \cos \alpha
\end{array}\right]
$$

As we have seen, there is a linear relationship between the experimentally determined strain projection $\epsilon_{\alpha}$ and the strain components in the sample coordinates $\epsilon_{i j}$, where the linear coefficients $q_{i}$ are simply the components of the scattering vector, meaning that:

$$
\epsilon_{\alpha}=q_{i} q_{j} \epsilon_{i j}
$$

Now the $q_{i}$ 's depend only on sample geometry parameters and Debye coordinates $\psi_{0}, \phi_{0}, \eta, \alpha$ and are thus known, so equation (22) represents a set of linear equations for each polar angle $\alpha$ at which the strain is determined. The four unknowns are the $\epsilon_{i j}$ (in plane stress $\epsilon_{13}=\epsilon_{23}=0$ ) and the independent terms $\epsilon_{\alpha}$ are determined experimentally. Since the number of $\alpha$ values probed is more than four (typically 360 at least), the system is overdetermined and can be numerically solved in the least-squares sense.

Experimentally, the Debye ring is fitted to obtain $2 \theta(\alpha)$ and then the strain is calculated using:

$$
\epsilon_{\alpha}=\ln \left(\frac{\sin \theta_{0}}{\sin \theta(\alpha)}\right)
$$

\subsection{Effect of diffraction volume}

For diffraction to occur, the incident and diffracting beam vectors and the normal to the diffracting planes must be co-planar, with the normal of the diffracting planes making equal angles with the two wavevectors. Consequently distinct groups of grains, with parallel diffracting plane normals, contribute to the diffraction profiles obtained at the various $\psi, \phi, \eta, \alpha$ coordinates. We define the total volume of such a set of grains as the information 
volume for these angular coordinates. All formulations discussed above for computing the stresses from diffraction data assume that the same quasi-homogeneous bi-axial stress tensor exists within all diffracting volumes sampled by all techniques. This is not a strong assumption and must be verified experimentally on a case-by-case basis. For example, this assumption is justified in shot-peened samples where, because of the random plastic deformation field caused by multiple impacts, any pre-existing crystallographic texture is destroyed in the peened layer, and the same macrostress distribution is obtained from all reflections and all accessible $\psi$ tilts of a given reflection. However, this is not the case for samples plastically deformed in uniaxial tension [8]. In such cases, the results from the three formulations might not agree.

\section{Materials and Methods}

\subsection{Experimental setup}

A miniature portable 2D x-ray apparatus (Pulstec $\mu$-X360, Pulstec Corp., Japan) was used to determine residual stresses in carbon steel tubular samples as a function of applied load. This apparatus has been described elsewhere [10]; in brief, it consists of a low intensity Cr $K_{\alpha}$ source with a filter and an image plate $30 \mathrm{~mm}$ in radius positioned normal to the incident beam. Under these conditions, back-diffracted beams from the sample surface are captured by the image plate at $50 \mu \mathrm{m}$ resolution. Beam spot size is approximately 2 $\mathrm{mm}$ for the default $1 \mathrm{~mm}$ pinhole collimator, and angular coverage for a $40 \mathrm{~mm}$ sample to detector distance is $2 \theta=145^{\circ}-175^{\circ}$.

An Instron 5984 Universal Testing Machine was used to apply a tensile load to the specimens via pneumatic grips. The machine was operated in load control and strain was recorded using a clip-on extensometer. Nominal loads corresponding to stresses in the range of $0-275 \mathrm{MPa}$ were applied in $25 \mathrm{MPa}$ increments, and at each load one x-ray diffraction pattern was acquired. Once a target load was reached it was held for the total duration of the x-ray measurement, which in our case amounted to $40 \mathrm{~s}$ of exposure plus $50 \mathrm{~s}$ of image plate readout and processing time.

\subsection{Samples}

Samples studied in this work were seamless tubes $25.4 \mathrm{~mm}$ in diameter and with a wall thickness of $1.24 \mathrm{~mm}$, conforming to ASTM 179 and SAE J524 standards. The tube material was very low carbon steel conforming to AISI 1010 specifications, which was cold drawn and heat treated at $650{ }^{\circ} \mathrm{C}$. The microstructure of this material is presented in Figure 3: as expected from the composition, it consisted of primary ferrite with small, isolated islands of pearlite. Ferrite grain size was determined to be $(12 \pm 2) \mu \mathrm{m}$ by the line intercept method.

Samples were checked for texture and phase composition by using a laboratory diffractometer using $\mathrm{Cu}$-radiation with a graphite monochromator (figure S1 of the supplementary information): no significant texture was detected (see figure S2). Due to the very low cementite content, no $\mathrm{Fe}_{3} \mathrm{C}$ peaks were observed. The use of tubular samples introduced an additional set of alignment difficulties, when compared with the more typical flat specimens, that were in our opinion more representative of actual applications where complex geometries, tight space constraints and alignment difficulties are to be expected (see Figure S3 in the supplementary information for actual pictures of the setup).

[Figure 3 about here.] 


\subsection{Data analysis and stress calculation}

Internal software supplied by the x-ray apparatus was used to compute detector parameters (beam center and sample-to-detector distances) at zero external load. This approach referred all subsequent measurements to this datum and ensured that, as long as the applied load stayed below the elastic limit (and, consequently, did not change any initial residual stresses), subsequent x-ray analysis would only yield the (uniaxial) applied stress values. Nominal detector-to-sample distances were in the $35-50 \mathrm{~mm}$ range, which resulted in only one Debye ring, from the (211) reflection of the BCC ferrite phase, being recorded. We used $\mathrm{a}=2.8664 \AA$ as the unstressed lattice parameter at zero load for calculating the sample to detector distance.

Detector tilt and/or distortion were determined by fitting the Debye ring to an ellipse using the hypothesis that, for a residual stress-free sample in the absence of applied load, any ellipticity in the Debye ring must be due to detector rotation. Since a deviatoric strain tensor would also result in a deformed, elliptical Debye ring, we fitted the rings obtained at zero applied stress using the full-ring procedure described previously and obtained a 'fictitious strain' (the strain state that would result in the same deformation of the Debye ring as observed due to detector tilt) that was used to correct subsequently determined strains under load.

For all stress calculations, the x-ray elastic constant, $\frac{1+\nu}{E}$, was taken to be $5.71 \mathrm{TPa}^{-1}$. This value was computed from the quasi-isotropic elastic parameters for ferrite, $E=$ $224 \mathrm{GPa}$ and $\nu=0.28$. Utilization of Neerfeld-Hill or Kroener x-ray elastic constants, computed from single-crrystal compliances for the (211) ferrite reflection ( 5.94 and $6.21 \mathrm{TPa}^{-1}$, respectively) would have uniformly shifted all calculated stress values by approximately $4 \%$ and $8 \%$. Since the term $\frac{1+\nu}{E}$ is a multiplier in all three formalisms, comparing the equivalency of their stress output is independent of the selection of elastic constants.

Two sets of data were acquired for each of the three different beam incidence angles studied, at $\psi_{0}=0^{\circ}, 35^{\circ}, 45^{\circ}$. This allowed us to study the effect of beam incidence angles on stress calculation, and also to obtain a set of strain values at different $\psi$ so as to be able to use the $\sin ^{2} \psi$ method for comparison. Nominal $\psi$ values studied were 11.8, 23.2, $33.2,46.8$ and $56.8^{\circ}$; additionally the extensometer provided the strain at $\psi=90^{\circ}$.

A code solving equation (22) in the least squares sense was implemented in MATLAB. A slight modification of the fitting equation was performed so the variation of scattering angle $\theta(\alpha)$ with the polar coordinate was performed directly without requiring the specification of a stress-free reference angle $\theta_{0}$ :

$$
\ln (\sin \theta(\alpha))=\ln \left(\sin \theta_{0}\right)-q_{i} q_{j} \epsilon_{i j}
$$

This has the advantage that the stress-free angle $\theta_{0}$ is now the independent term and can thus be obtained as part of the least-squares fit. The previous equation is, in theory, valid for the general case of a triaxial stress state, however some simplifications can be introduced for plane stress conditions, namely $\epsilon_{13}=\epsilon_{23}=0$ and $\epsilon_{33}=-\nu\left(\epsilon_{11}+\epsilon_{22}\right)$, where $\nu$ is the Poisson's ratio of the material.

The produced code was tested by generating synthetic data sets, adding normal noise and then fitting the resulting data to obtain the stress components and compare with the initial values. Additionally, the impact of errors in the determination of some of the input parameters (sample rotation) in the final stress result was assessed by means of these simulations. 


\section{Results and Discussion}

Figure 4 shows the raw information from the image plate, showing some spottiness of the Debye ring, also evidenced in the variation of the (211) Fe peak area along the Debye ring and, to a lesser extent, in the change in FWHM of the peak. There are also variations in the experimental $2 \theta$ vs $\alpha$ profiles as can be seen in figure 5 , which shows the $2 \theta$ position of the peaks as a function of $\alpha$, along the Debye ring, for tests performed at $\psi_{0}=35^{\circ}, 45^{\circ}$. The measured profiles exhibit slight systematic undulations around the expected values: these 'undulations' are along the $\alpha$ angle and are not related to the well-known oscillations in $\sin ^{2} \psi[22-26]$, but are due to the (proprietary) pixel intensity-data analysis algorithm in this particular machine; these algorithms are inaccessible to the users. We also observed these undulations in data measured from the calibration powders (annealed ferrite powder with grain size around $1 \mu \mathrm{m}$ ) supplied by the system manufacturer [10]. Peak area plots as a function of azimuthal angle (Figure 4, lower left panel) were uniform and allowed us to discount effects of crystallographic texture.

[Figure 4 about here.]

[Figure 5 about here.]

As we saw in section 2.3 (and Figure 2), a single exposure that covers the whole Debye ring also allows for the determination of stress using the $\sin ^{2} \psi$ method, although only using the two points that correspond to $\phi=0$, namely those at $\alpha=0,180^{\circ}$. In this case the information volume of the two-tilt $\sin ^{2} \psi$ method is a subset of the information volume of the Debye ring analysis. In light of this fact, we used diffraction data from a single exposure to calculate the average axial stress and standard deviation using both area detector-based single exposure techniques, and the two-tilt $\sin ^{2} \psi$ method. This approach somewhat alleviated the sampling issues arising from the use of multiple $\psi$ tilts, with completely independent information volumes. A comparison between the $\sin ^{2} \psi$ and the full Debye ring method can be seen in Figure 5: in addition to experimental Debye ring profiles at different stresses, we plot simulated Debye rings where the input stresses value is obtained from the $\sin ^{2} \psi$ method. Reasonable agreement is observed.

Figure 6 shows axial $\left(\sigma_{x x}\right)$ stresses calculated using single exposure methods (either least squares fitting of the entire Debye ring or $\cos \alpha$ ) against stresses calculated using the conventional (two-tilt) $\sin ^{2} \psi$ approach. Black symbols represent averages over four measurements at $\psi_{0}=35,45^{\circ}$ and error bars represent \pm one standard deviation. For completeness, individual measurements are plotted as well, using red symbols (hollow for $\psi_{0}=35^{\circ}$ and filled for $\psi_{0}=45^{\circ}$ ). The dashed line, with slope of 1 , denotes the locus of identical values. The following observations can be made:

1-The precision of 'real' residual stress measurements on an enginering sample, obtained under conditions which are similar to those encountered during typical field practice, is worse, by an order of magnitude, than the values quoted for 'ideal' samples, i.e, \pm 20 vs. $\pm 2 \mathrm{MPa}$. This is consistent with past residual-stress measurement practice using laboratory and field instruments. We again note that the scatter in the measured data in this figure is related to the precision (not accuracy) of the particular system (instrument, sample, measurement conditions).

2-The stress values obtained from both area-detector based single exposure techniques are, within error, equal to the two-tilt $\sin ^{2} \psi$ formulation for applied stresses in the elastic range (Figure 7). For higher applied stresses we observe a systematic deviation of the stresses measured by the single exposure techniques from the dashed line denoting 1-to1 correspondence with the two-tilt $\sin ^{2} \psi$ formulation. As noted before, this deviation 
might be caused by either (i) the elastic grain interaction stresses forming in response to the heterogeneous distribution of plastic flow in the microstructure, and the correspondent heterogeneous elastic residual strain distribution within the diffracting volumes, or (ii) the modification of any existing residual stresses due to plastic flow, or a combination of both effects [5]. This point should be taken into consideration in the selection of instruments/techniques for measurements on samples with complex residual stress distributions, as well as in the interpretation of the results. It might be possible to overcome these issues with careful texture measurements, calibrated elastic constants and a better theoretical framework. We are working on this issue and will report our findings in a later article.

[Figure 6 about here.]

[Figure 7 about here.]

\section{Error analysis}

Even though errors associated with the $\sin ^{2} \psi$ method are extensively treated in the literature $[8,9]$, there are very few publications dealing with the errors associated with single-tilt, area-detector-based, techniques. We used the equations presented in Section 2 to investigate the sensitivity of $\cos \alpha$ and full Debye ring fitting formalisms to variations in measurement parameters, such as $2 \theta$ values vs. $\alpha$. We chose to study errors associated with the angle $\psi_{0}$ because it will be difficult to measure accurately for a portable detector not mounted in a goniometer with precise reference to the sample. For this purpose, we performed a series of calculations aimed to assess the impact of errors in the Debye ring radius determination $\Delta(2 \theta)$ as well as error in sample rotation $\Delta \psi_{0}$. For the first case, gaussian noise was added to the Debye rings with various standard deviations. For each selected standard deviation, a total of 200 Debye rings were simulated with a uniaxial stress of $\sigma_{x x}=0$ and fitted (see figure 8 for an example), and the standard deviation of the calculated stress was computed. Results are shown in the top panel of figure 9. Both methods gave almost identical results, and it is clear from the data that both are robust: if errors in $2 \theta$ were normally distributed (although admittedly, they usually are not) then only a standard deviation of $10^{-2}$ would be required to determine uniaxial stress with a relative error of $10 \%$.

The errors in determination of the sample orientation have a much higher impact. To assess this effect, Debye rings were calculated again for a uniaxial stress of $\sigma_{x x}$ and different orientations $\psi_{0}+\Delta \psi_{0}$, and later fitted with both methods using the 'wrong' value $\psi_{0}$. The bottom panel of figure 9 shows our results: the error in stress determination is larger for larger $\delta \psi_{0}$ as expected, but also for a given $\delta \psi_{0}$ it is larger for smaller $\psi_{0}$, meaning that accurate measurements require large sample tilts which are less sensitive to misalignment. Unfortunately, the irradiated area on the sample becomes much larger at higher $\psi_{0}$ tilts; which can cause defocussing errors. Further, this will limit the use of the technique to strain fields which are homogeneous over larger areas, and away from features such as fillets, weld-spots, key-ways, etc. which can cause rapid stress variations.

[Figure 8 about here.]

[Figure 9 about here.] 


\section{Conclusions}

We have rigorously compared the methods used for determining residual stresses from diffraction profiles acquired with a portable x-ray measurement device utilizing an area detector. Our results show that the $\cos \alpha$ and the direct least squares fitting method for the entire Debye ring are theoretically identical to the two-tilt $\sin ^{2} \psi$ technique for a biaxial stress state which is homogeneous in the diffraction volumes of the three techniques. Our experimental results using in-situ uniaxial loading verify this conclusion. We observe, however, that plastic flow during loading impairs this equivalency. Consequently, we do not recommend the application of these techniques to samples possessing general strain tensors, or heterogeneous elastic strain distributions without careful calibration and analysis.

\section{Acknowledgments}

Loading experiments were performed at the Robert W. Carleton Strength of Materials Laboratory, Columbia University. Dr. A. Brügger's assistance with the loading setup is gratefully ackonowledged. The x-ray portable stress measurement device was kindly supplied by Pulstec Industrial Co., Ltd. Authors would like to thank Toshikazu Suzuki and Yoshinobu Teramoto for installation and technical support. J. Ramirez-Rico gratefully acknowledges support from the Universidad de Sevilla Research Fund (V Plan Propio).

\section{Compliance with Ethical Standards}

The authors declare that they have no conflict of interest.

\section{References}

[1] K. Heindlhofer, Evaluation of Residual Stress. New York: McGraw-Hill Book Co., 1948.

[2] C. S. Barrett, Structure of Metals. New York: McGraw-Hill Book Co., 1952.

[3] A. Taylor, X-ray Metallography. New York: John Wiley and Sons, 1961.

[4] R. J. Homicz, "Fundamentals and basic techniques of residual stress measurements with a portable x-ray diffraction unit," Society of Automotive Engineers Transactions, vol. 76, no. s1, pp. 912-917, 1967.

[5] M. R. James and C. J. B, "The measurement of residual stresses by x-ray diffraction techniques," Treatise on Materials Science and Technology, vol. 19, no. A, pp. 1-62, 1978.

[6] M. Gelfi, E. Bontempi, R. Roberti, and L. Depero, "X-ray diffraction debye ring analysis for stress measurement (drast): a new method to evaluate residual stresses," Acta Materialia, vol. 52, no. 3, pp. 583-589, 2004.

[7] B. B. He, Two-dimensional X-ray diffraction. John Wiley \& Sons, 2011.

[8] I. C. Noyan and J. B. Cohen, Residual Stress. New York: Springer-Verlag, 1987.

[9] V. Hauk, Structural and residual stress analysis by nondestructive methods: Evaluation-Application-Assessment. Elsevier, 1997. 
[10] J. Ling and S.-Y. Lee, "Characterization of a portable x-ray device for residual stress measurements," Advances in X-ray Analysis, vol. 59, pp. 153-161, 2015.

[11] B. Boyce, T. Furnish, H. Padilla II, D. Van Campen, and A. Mehta, "Detecting rare, abnormally large grains by x-ray diffraction," Journal of Materials Science, vol. 50, no. 20, pp. 6719-6729, 2015.

[12] J. Ramirez-Rico, F. Stolzenburg, J. Almer, J. Routbort, D. Singh, and K. Faber, "In situ imaging and strain determination during fracture in a sic/sic ceramic matrix composite," Scripta Materialia, vol. 69, no. 7, pp. 497-500, 2013.

[13] B. J. Harder, J. Ramirez-Rico, J. D. Almer, K. N. Lee, and K. T. Faber, "Chemical and mechanical consequences of environmental barrier coating exposure to calciummagnesium-aluminosilicate," Journal of the American Ceramic Society, vol. 94, no. article 670151, pp. s178-s185, 2011.

[14] T. Sasaki, Y. Hirose, K. Sasaki, and S. Yasukawa, "Influence of image processing conditions of debye scherrer ring images in X-ray stress measurement using an imaging plate," Advances in X-ray Analysis, vol. 40, pp. 588-594, 1997.

[15] T. Sasaki and Y. Kobayashi, "X-ray multiaxial stress analysis using two debye rings," Advances in X-ray Analysis, vol. 52, pp. 248-255, 2009.

[16] T. Sasaki, Y. Maruyama, H. Ohba, and S. Ejiri, "Two-dimensional imaging of debyescherrer ring for tri-axial stress analysis of industrial materials," Journal of Instrumentation, vol. 9, pp. C07066, 2014.

[17] A. Kampfe, B. Kampfe, S. Goldenbogen, B. Eigenmann, E. Macherauch, and D. Lohe, "X-ray stress analysis on polycrystalline materials using two-dimensional detectors," Advances in X-ray Analysis, vol. 43, pp. 54-65, 2000.

[18] H. Behnken and V. Hauk, "Determination of steep stress gradients by x-ray diffraction - results of a joint investigation," Materials Science and Engineering: A, vol. 300, no. 1, pp. 41-51, 2001.

[19] M. Marques, A. Dias, P. Gergaud, and J. Lebrun, "A methodology development for the study of near surface stress gradients," Materials Science and Engineering: A, vol. 287, no. 1, pp. 78-86, 2000.

[20] H. Dolle, "The influence of multiaxial stress states, stress gradients and elastic anisotropy on the evaluation of (residual) stresses by x-rays," Journal of Applied Crystallography, vol. 12, no. 6, pp. 489-501, 1979.

[21] S. Taira, K. Tanaka, and T. Yamazaki, "A method of x-ray microbeam measurement of local stress and its application to fatigue crack growth problems," Journal of the Society of Materials Science, Japan, vol. 27, no. 294, pp. 251-256, 1978.

[22] D. Chidambarrao, Y. Song, and I. Noyan, "Numerical simulation of the X-ray stress analysis technique in polycrystalline materials under elastic loading," Metallurgical and Materials Transactions A, vol. 28, no. 12, pp. 2515-2525, 1997.

[23] I. Noyan and L. Nguyen, "Oscillations in interplanar spacing vs. $\sin 2 \psi$ a fem analysis," Advances in X-Ray Analysis, vol. 31, pp. 191-204, 1988. 
[24] I. Noyan and L. Nguyen, "Effect of plastic deformations on oscillations in $d$ vs. $\sin ^{2} \psi$ plots," Advances in X-Ray Analysis, vol. 32, pp. 355-364, 1989.

[25] I. Noyan and J. Cohen, "Determining stresses in the presence of nonlinearities in interplanar spacing vs. $\sin ^{2} \psi, "$ Advances in X-Ray Analysis,, vol. 27, pp. 129-148, 1983.

[26] V. Hounkpati, S. Fréour, D. Gloaguen, and V. Legrand, "Influence of morphologic texture on stress analysis by x-ray and neutron diffraction in single-phase metallic materials," Journal of Materials Science, vol. 49, no. 20, pp. 7049-7065, 2014. 


\section{List of Figures}

1 Schematic geometry used for the experiments and analysis perfomed in this work . . . . . . . . . . . . . . . . . . 15

2 Stereographic projection sample coordinates $\left(S_{1}, S_{2}, S_{3}\right)$ for the case of sample rotations with $\omega_{0}=\phi_{0}=0$. In this case the scattering vectors laying at $\alpha=0, \pi$ correspond to angles $\psi=\psi_{0} \pm \eta$ in sample coordinates. . . . . 16

3 Microstructure of the 1010 carbon steel samples studied in this work: optical microscopy (left) and scanning electron microscopy (right). . . . . . . . . 17

4 Raw data from the image plate at zero applied stress, for the case of $\psi_{0}=35^{\circ}$. Top: normalized diffraction intensity as a function of $2 \theta$ and $\alpha$. Bottom-left: peak area along the Debye ring. Bottom-right: peak fwhm along the Debye ring. . . . . . . . . . . . . . . . . . . 18

5 Debye ring radius $(2 \theta)$ as a function of azimuthal angle $\alpha$ for the ferrite (211) reflection, at different loads. Symbols correspond to experimental data while solid lines are calculated Debye ring profiles using the stress obtained from the $\sin ^{2} \psi$ method. . . . . . . . . . . . . . . 19

6 Stresses calculated from single exposures (full-2d, $\cos \alpha$ vs $\sin ^{2} \psi$ stress. Black symbols represent averages over four measurements at $\psi_{0}=35,45^{\circ}$ and error bars represent one standard deviation. Red symbols (hollow for $\psi_{0}=35^{\circ}$ and filled for $\psi_{0}=45^{\circ}$ ) correspond to individual measurements. . 20

7 Uniaxial tensile test data of the 1010 steel tube sample used in the study. The extrapolated elastic stress line is calculated from least-squares fitting of the 0-50 MPa load range, which yielded $210 \mathrm{GPa}$ as the (effective) Young's Modulus. The $\Delta \sigma$ values (right ordinate) show the difference between the extrapolated elastic stress and the actual load cell output. . . . . . . . . 21

8 Simulated Debye rings and fitting results for the full-ring fitting method, after adding gaussian noise. . . . . . . . . . . . . . . . 22

9 Top: Error in the determination of stress as a function of error in $2 \theta$ (as one standard deviation). The hollow symbols are for the full-ring fitting method while the filled symbols are for the $\cos \alpha$. Bottom: stress calculated as a function of error in sample orientation (see text for details), for a uniaxial stress state with $\sigma_{x x}=100 \mathrm{MPa}$ and for different $\psi_{0}$ values. . . . . . . . 23 


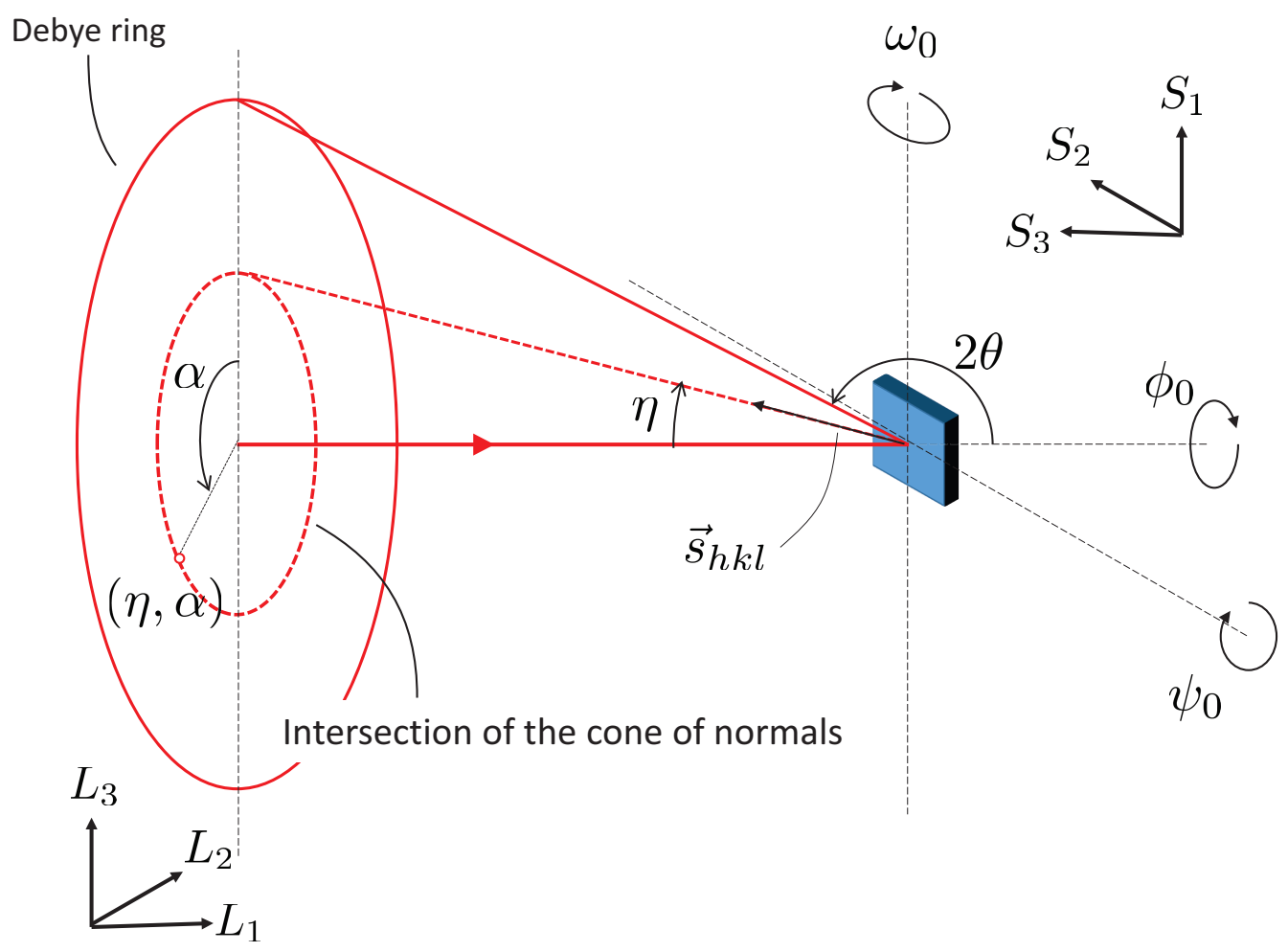

Figure 1: Schematic geometry used for the experiments and analysis perfomed in this work 


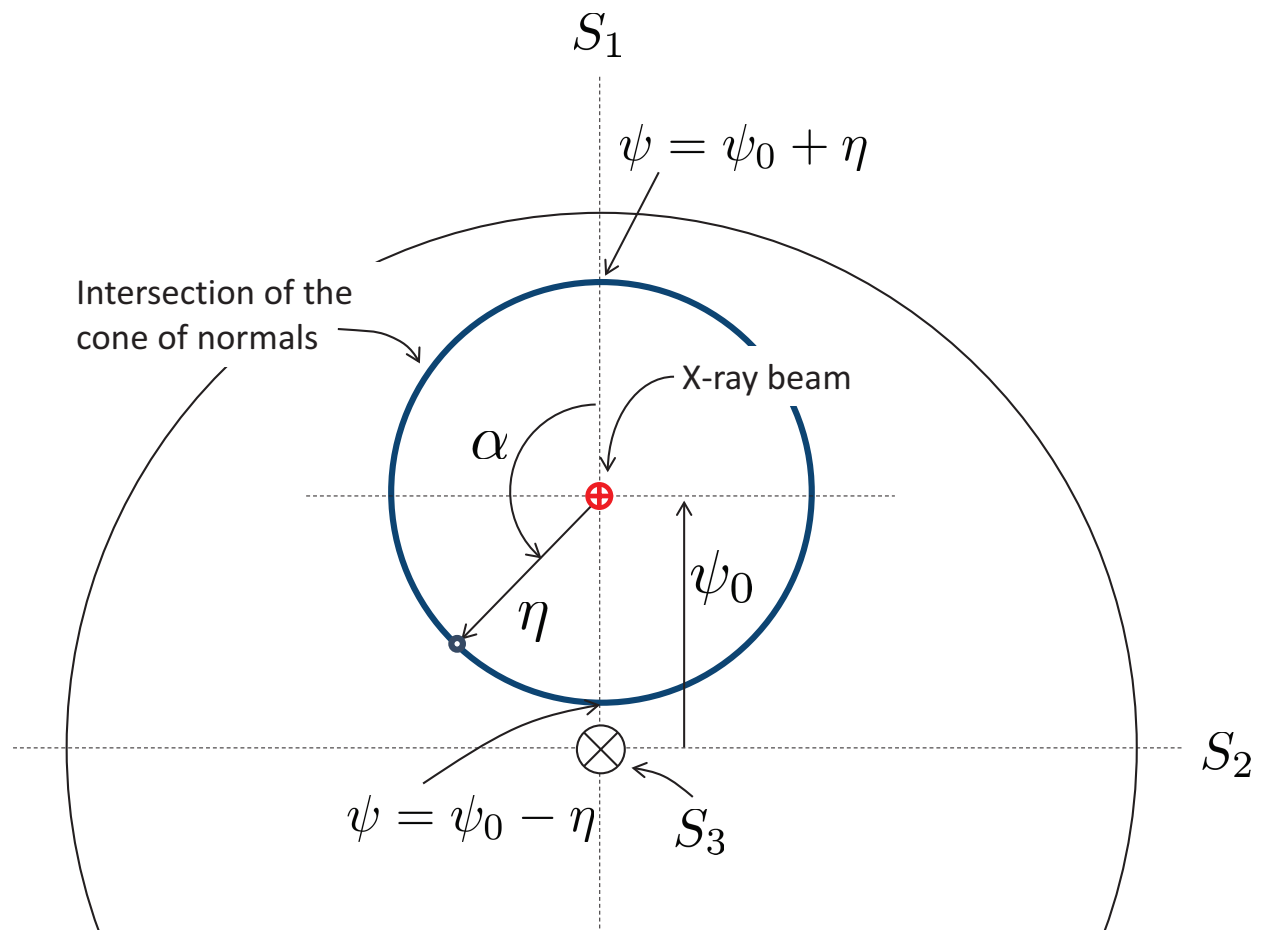

Figure 2: Stereographic projection sample coordinates $\left(S_{1}, S_{2}, S_{3}\right)$ for the case of sample rotations with $\omega_{0}=\phi_{0}=0$. In this case the scattering vectors laying at $\alpha=0, \pi$ correspond to angles $\psi=\psi_{0} \pm \eta$ in sample coordinates. 

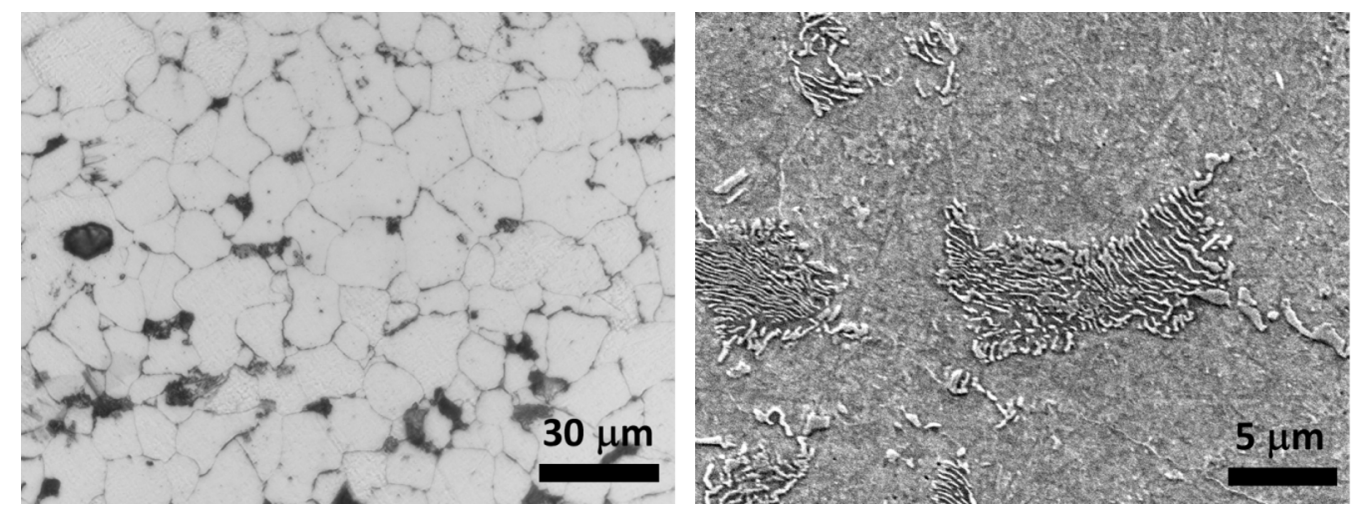

Figure 3: Microstructure of the 1010 carbon steel samples studied in this work: optical microscopy (left) and scanning electron microscopy (right). 

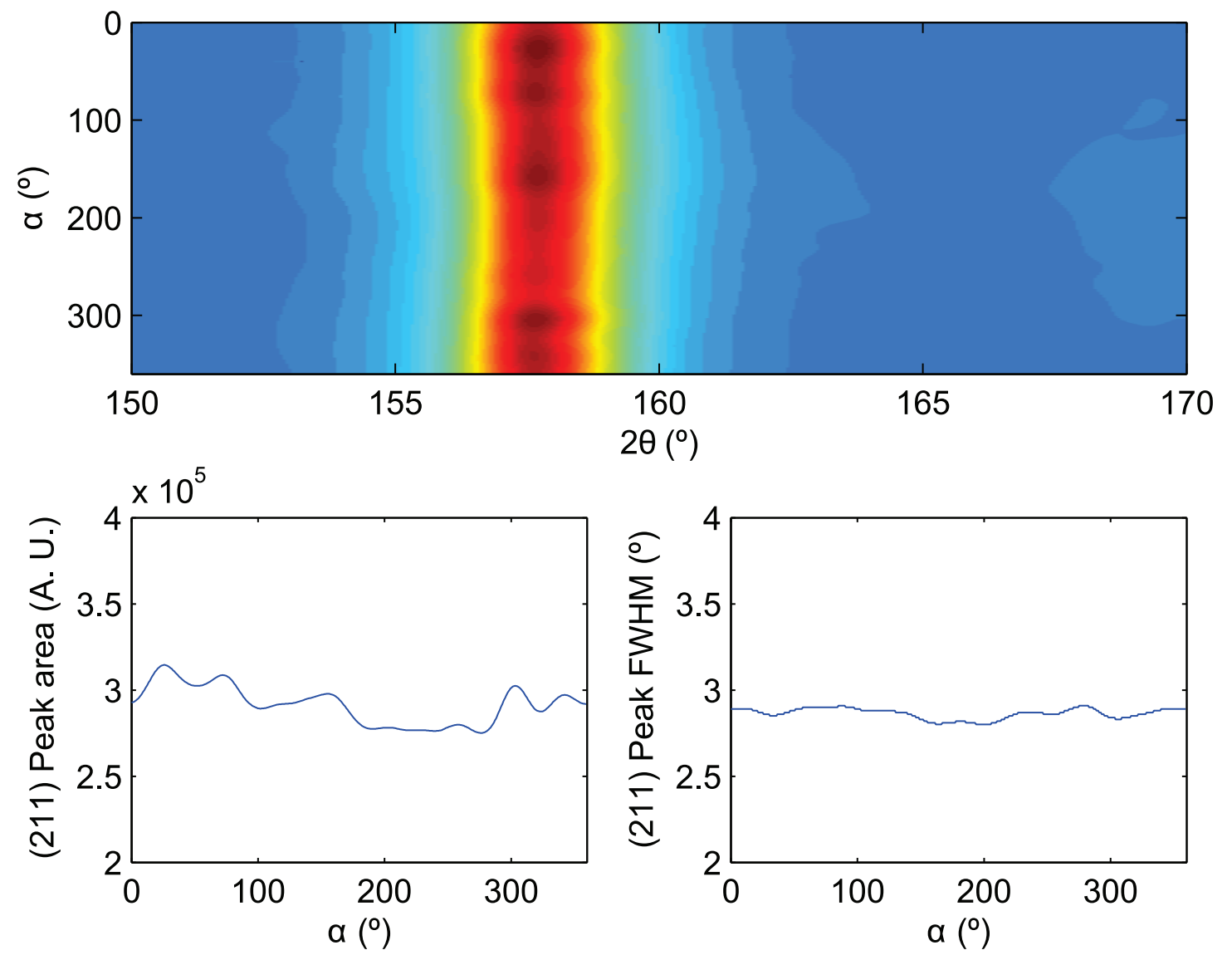

Figure 4: Raw data from the image plate at zero applied stress, for the case of $\psi_{0}=35^{\circ}$. Top: normalized diffraction intensity as a function of $2 \theta$ and $\alpha$. Bottom-left: peak area along the Debye ring. Bottom-right: peak fwhm along the Debye ring. 

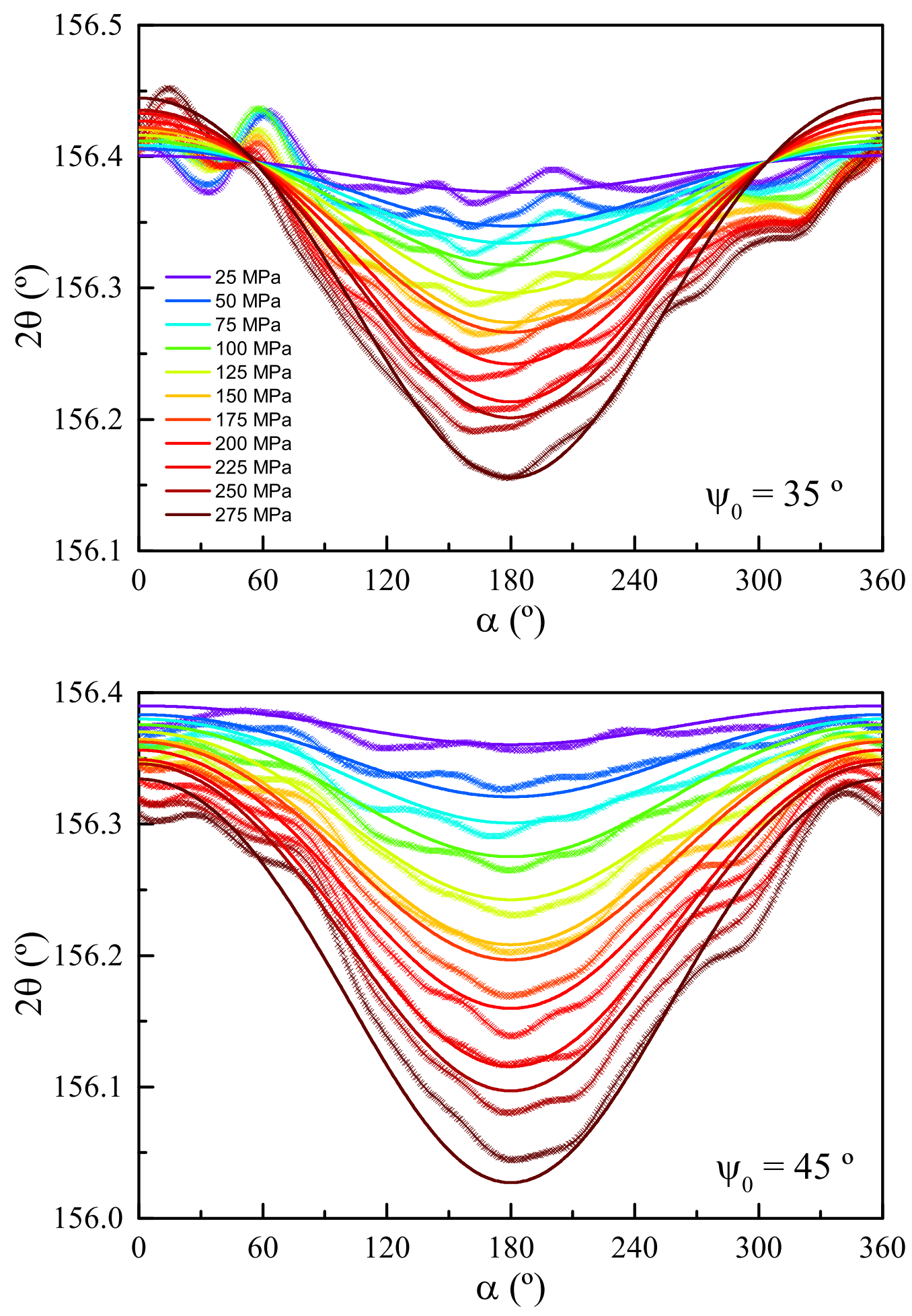

Figure 5: Debye ring radius $(2 \theta)$ as a function of azimuthal angle $\alpha$ for the ferrite (211) reflection, at different loads. Symbols correspond to experimental data while solid lines are calculated Debye ring profiles using the stress obtained from the $\sin ^{2} \psi$ method. 


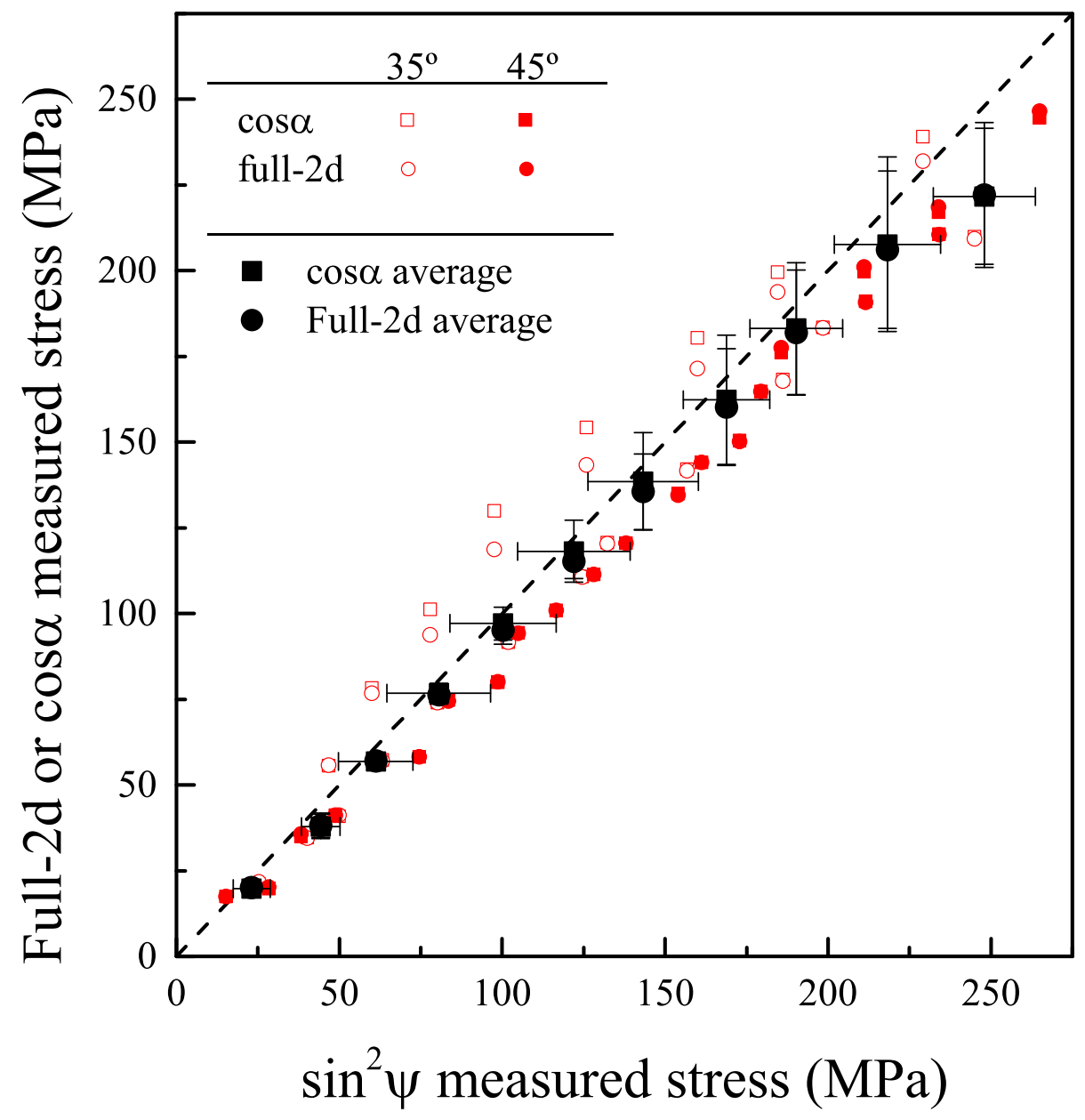

Figure 6: Stresses calculated from single exposures (full-2d, $\cos \alpha$ vs $\sin ^{2} \psi$ stress. Black symbols represent averages over four measurements at $\psi_{0}=35,45^{\circ}$ and error bars represent one standard deviation. Red symbols (hollow for $\psi_{0}=35^{\circ}$ and filled for $\psi_{0}=45^{\circ}$ ) correspond to individual measurements. 


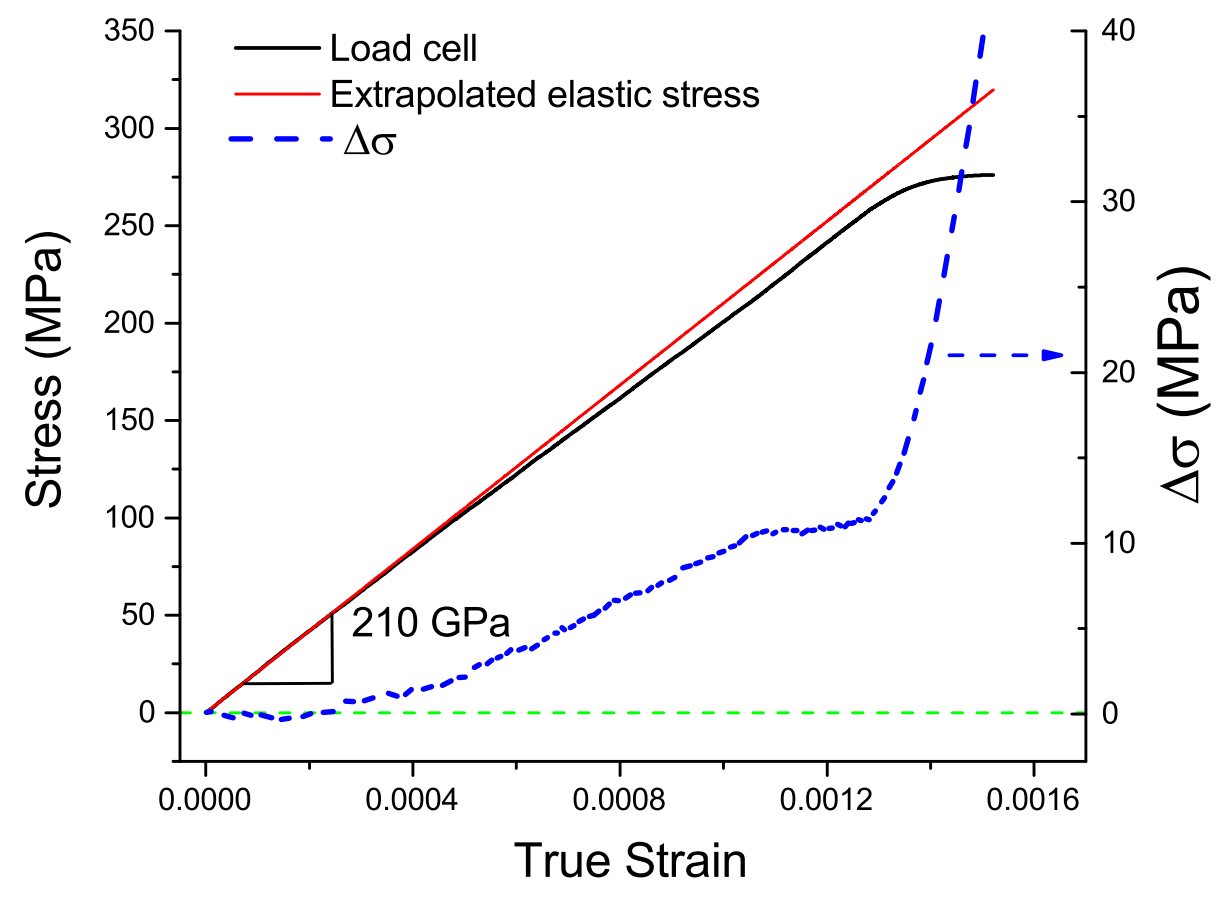

Figure 7: Uniaxial tensile test data of the 1010 steel tube sample used in the study. The extrapolated elastic stress line is calculated from least-squares fitting of the 0-50 MPa load range, which yielded $210 \mathrm{GPa}$ as the (effective) Young's Modulus. The $\Delta \sigma$ values (right ordinate) show the difference between the extrapolated elastic stress and the actual load cell output. 

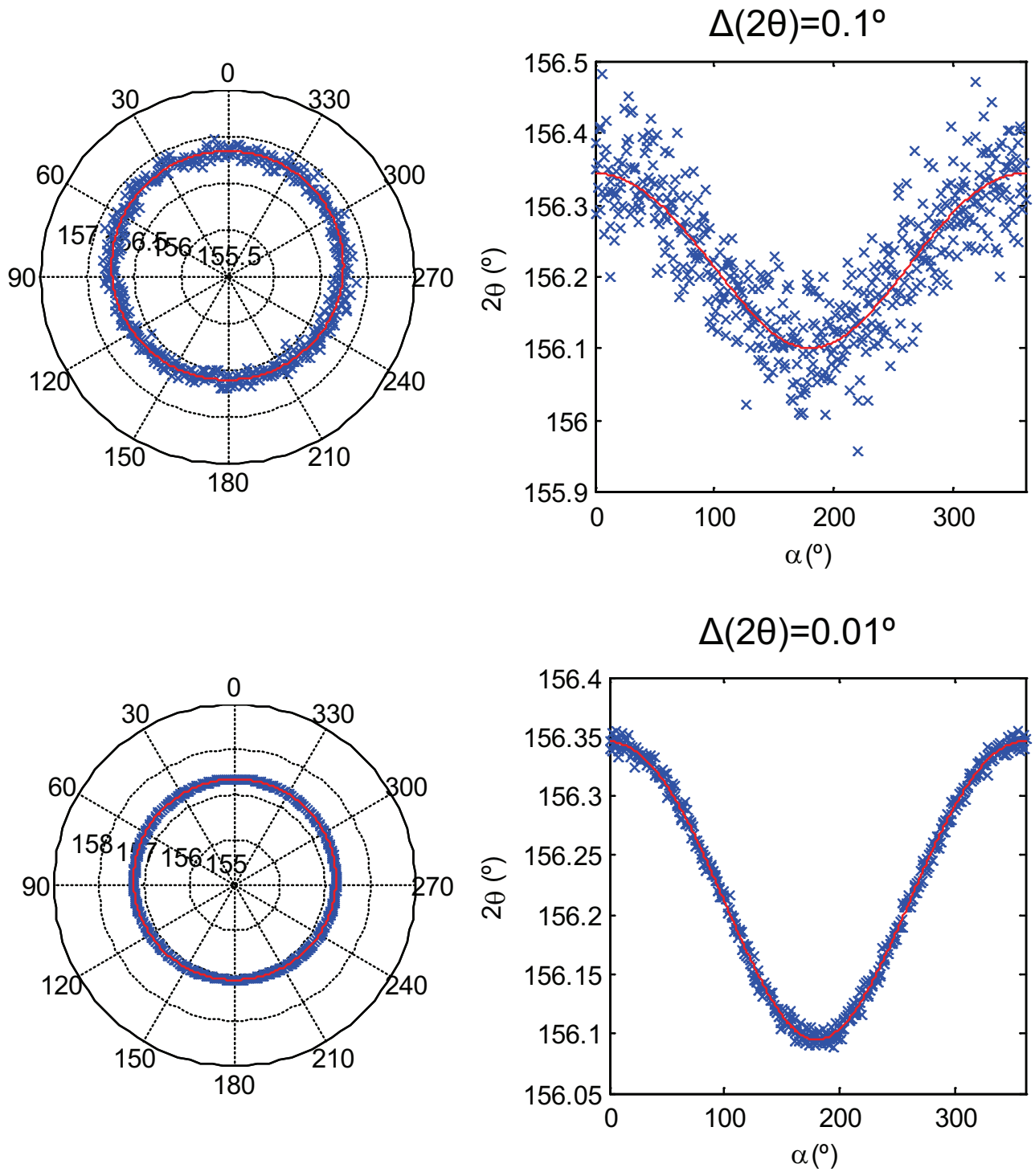

Figure 8: Simulated Debye rings and fitting results for the full-ring fitting method, after adding gaussian noise. 

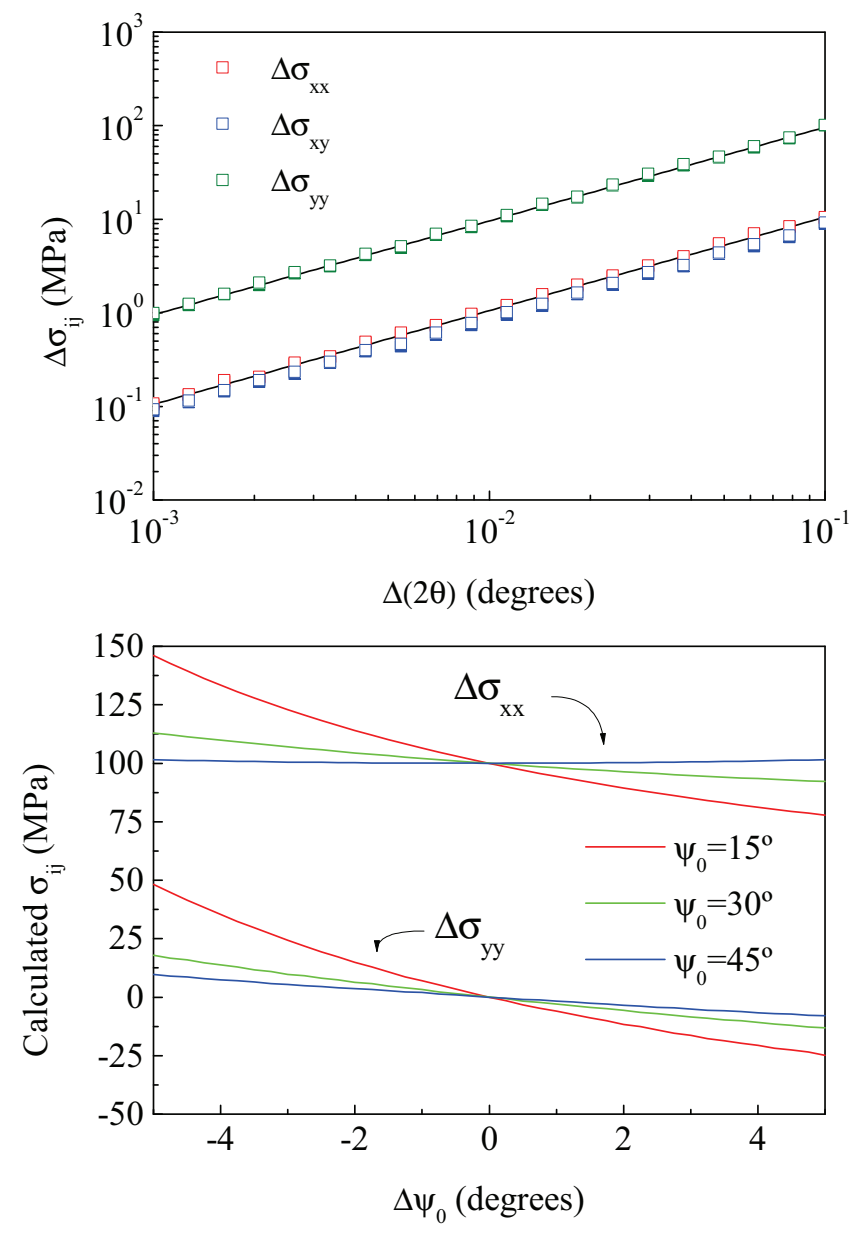

Figure 9: Top: Error in the determination of stress as a function of error in $2 \theta$ (as one standard deviation). The hollow symbols are for the full-ring fitting method while the filled symbols are for the $\cos \alpha$. Bottom: stress calculated as a function of error in sample orientation (see text for details), for a uniaxial stress state with $\sigma_{x x}=100 \mathrm{MPa}$ and for different $\psi_{0}$ values. 